\title{
Subgroups of Sjögren's syndrome patients categorised by serological profiles: clinical and immunological characteristics
}

\author{
Ewa Kontny ${ }^{1}$, Aleksandra Lewandowska-Poluch ${ }^{2}$, Magdalena Chmielińska², Marzena Olesińska² \\ ${ }^{1}$ Department of Pathophysiology and Immunology, National Institute of Geriatrics, Rheumatology and Rehabilitation, Warsaw, Poland \\ ${ }^{2}$ Clinic of Connective Tissue Diseases, National Institute of Geriatrics, Rheumatology and Rehabilitation, Warsaw, Poland
}

\begin{abstract}
Objectives: Sjögren's syndrome (SS) is an autoimmune disease characterised by heterogeneous clinical presentation and presence of various autoantibodies - anti-SSA/Ro of diagnostic value, less specific anti-SSB/La and others. We searched for biomarker(s) and potential therapeutic target(s) of SS subsets that vary in their autoantibody profile.

Material and methods: Eighty-one patients with SS (70 female and 11 male) and 38 healthy volunteers (28 female and 10 male) were included in the study. Patients were categorised according to absence (group 1) or presence of anti-SSA/Ro antibody which occurred either alone (group 2) or together with anti-SSB/La (group 3). Clinical evaluation was performed, and presence of autoantibodies and concentrations of cytokines relevant to SS pathogenesis, i.e. a proliferation inducing ligand (APRIL), B-lymphocyte activating factor (BAFF), interleukin (IL) 4, IL-10, interferon $\alpha$ (IFN- $\alpha$ ) and thymic stromal lymphopoietin (TSLP), in sera were determined.

Results: Frequency of autoantibodies other than anti-SSA/Ro and anti-SSB/La, the number of autoantibody specificities and anti-nuclear antibody titres were higher in group 2 and/or 3 than in group 1 of SS patients. Moreover, SS patients of groups 2 and 3 developed disease symptoms at younger age, and more often had positive Schirmer's test and skin lesions. In addition, serum concentrations of APRIL, but not other tested cytokines, were significantly higher in the patients of both groups 2 and 3 than those of group 1 and healthy volunteers.

Conclusions: Sjögren's syndrome patients with signs of B-cell epitope spreading are characterised by early disease onset, more frequent xerophthalmia and skin involvement, and up-regulated serum APRIL level. We suggest that therapeutic neutralisation of APRIL may be beneficial for these patients.
\end{abstract}

Key words: Sjögren's syndrome, clinical symptoms, epitope spreading, APRIL.

\section{Introduction}

Sjögren's syndrome (SS) is an autoimmune disease characterised by chronic inflammation of exocrine (mainly ocular and salivary) glands leading to their progressive destruction, severe xerophthalmia and xerostomia. Destruction of the exocrine gland epithelium, mediated by an abnormal immune response of $T$ and $B$ cells to autoantigens (Ro/SSA, La/SSB and others), is thought to be the major mechanism of SS development. The disorder occurs either in a primary (pSS) or secondary (sSS) form, with the latter accompanying other autoimmune diseases, pointing to differences in the pSS and sSS pathology. The clinical presentation of SS is heterogeneous and varies from sicca symptoms to systemic disease. Extra-glandular manifestations can occur in about one third of cases and some of these symptoms (e.g. palpable purpura, cryoglobulinaemia, non-Hodgkin's lympho$\mathrm{ma}$ ) are associated with increased mortality [1-3].

Diagnosis of the syndrome is based on the detection of autoantibodies in sera samples and histological

Address for correspondence:

Ewa Kontny, Department of Pathophysiology and Immunology, National Institute of Geriatrics, Rheumatology and Rehabilitation,

1 Spartańska St., 02-637 Warsaw, Poland, e-mail: ewa.kontny@spartanska.pl

Submitted: 21.08.2018; Accepted: 30.11.2018 
analysis of salivary gland tissue biopsy. According to recently developed ACR/EULAR classification criteria for pSS, either salivary gland biopsy or anti-Ro antibody must be positive to establish the diagnosis, while positivity for antinuclear antibodies (ANA), rheumatoid factor (RF) or isolated anti-La antibody presence is excluded because of a lack of specificity [4]. These new criteria also include extra-glandular symptoms and B cell activation markers that reflect the systemic nature of the syndrome [4]. Thus, although SS is characterised by the presence of a variety of autoantibodies, only antiSSA/Ro have diagnostic value. Despite this, there are data showing associations between the different autoantibody specificities encountered in SS and clinical symptoms, suggesting contribution of various autoantibodies to disease pathology [5].

Disease-related autoantibodies are directed against components of the human Ro/La ribonucleoprotein (RNP) complex, which is composed of small uridine-rich cytoplasmic RNAs and covalently associated proteins of different biological functions - R052, Ro60 and La. The Ro52 antigen is E3 ligase that provides negative feedback regulation in inflammation, Ro60 is an RNA-degrading protein while La is a phosphoprotein that among other cellular functions regulates RNA availability [6]. Interestingly, autoantibodies occur as early as up to 18-20 years before the pSS diagnosis, and ANAs, followed by RF, antiSSA/Ro and anti-SSB/La, are found more often [7]. In established disease anti-SSA/Ro and anti-SSB/La antibodies are present in $33-74 \%$ and $23-52 \%$ of pSS patients, respectively. Usually, anti-SSA/Ro antibodies are found either alone or concomitantly with anti-SSB/La, whereas anti-SSB/La very rarely exist in isolation [5]. It is suggested that the serological profile may be useful in the identification of patients who require more aggressive treatment, including suitable biological drugs [8].

The present study focused on searching for biomarker(s) and potential therapeutic target(s) of SS subsets that vary in an autoantibody profile. To this aim we compared clinical and immunologic features of SS patients categorised into 3 serological subgroups: 1) patients with neither anti-SSA/Ro nor anti-SSB/La antibodies, 2) patients positive for anti-SSA/Ro antibody only, and 3) patients seropositive for both anti-SSA/Ro and anti-SSB/La antibodies. Immunologic evaluation included the measurement of serum concentrations of cytokines that support a humoral response, i.e. a proliferation inducing ligand (APRIL), B-lymphocyte activating factor (BAFF), interleukin (IL-) 4, and IL-10, or cytokines that are suggested to contribute to other aspects of SS pathology and to be associated with disease activity, i.e. interferon $\alpha(\mathrm{IFN}-\alpha)$ and thymic stromal lymphopoietin (TSLP) [9-13].

\section{Material and methods}

Eighty-one patients suffering from Sjögren's syndrome (70 female [F] and 11 male [M]; median [range] age $=56.5$ [29-77]) and 38 healthy volunteers (28 F/10 M; median [range] age $=48$ [30-67]) were included in the study.

Table I. Patients' demographic, clinical and serological features

\begin{tabular}{|c|c|}
\hline Patients $(n)$ & 81 \\
\hline primary/secondarya Sjögren's syndrome & $77.2 / 22.8$ \\
\hline \multicolumn{2}{|l|}{ Demographics } \\
\hline Age, median (range), years & $56.5(29-77)$ \\
\hline Gender, female (F)/male (M), $n$ & $70 \mathrm{~F} / 11 \mathrm{M}$ \\
\hline Age at diagnosis, median (range), years & $47(18-82)$ \\
\hline Disease duration, median (range), months & $24(0-570)$ \\
\hline \multicolumn{2}{|l|}{ Clinical symptoms (\%) } \\
\hline Xerostomia & 95 \\
\hline Xerophthalmia & 93.7 \\
\hline Ocular test positive ${ }^{b}$ & 57.7 \\
\hline \multicolumn{2}{|l|}{ Focus score } \\
\hline 0 & 20.5 \\
\hline 1 & 36 \\
\hline 2 & 25.6 \\
\hline$\geq 3$ & 17.9 \\
\hline Extra-glandularc & 89.9 \\
\hline Systemic ${ }^{d}$ & 86 \\
\hline Abnormal laboratory test resultse & 89.9 \\
\hline Hypocomplementaemia (low C3 and/or low C4) & 35.4 \\
\hline Hypergammaglobulinaemia & 43 \\
\hline \multicolumn{2}{|l|}{ Autoantibodies (\%) } \\
\hline ANA positive & 97 \\
\hline Anti-SSA/Ro positive & 81.5 \\
\hline Anti-SSB/La & 48.2 \\
\hline RF & 51.9 \\
\hline Other $^{f}$ & 16.5 \\
\hline \multicolumn{2}{|l|}{ Medications (\%) } \\
\hline Antimalarial drugs & 56.2 \\
\hline Glucocorticoids & 62.9 \\
\hline
\end{tabular}

All values are $\%$ unless stated otherwise. ${ }^{a}$ Among the patients with secondary Sjögren's syndrome 54\% had rheumatoid arthritis, $25 \%$ systemic lupus erythematosus, $8.4 \%$ antiphospholipid syndrome, $8.4 \%$ systemic sclerosis and $4.2 \%$ mixed connective tissue disease; 'bShirmer's test results $\leq 5 \mathrm{~mm} / \mathrm{min}$; 'involvement of skin, lungs, kidney, nervous or musculoskeletal systems; dfever, weakness, lymphadenopathy or weight loss; 'leucopenia, lymphopenia, neutropenia, thrombocytopenia, increased serum concentration of C-reactive protein (CRP) or increased value of erythrocyte sedimentation rate (ESR); flupus anti-coagulant (LAC), autoantibodies specific to ribonucleoprotein (RNP), centromere, cardiolipin or $\beta 2$-glycoprotein. 
They were recruited from patients admitted to the Clinic of Connective Tissue Diseases of the National Institute of Geriatrics, Rheumatology and Rehabilitation (NIGRR) or NIGRR staff, respectively. Written informed consent was obtained from participants before they entered the study.

This study was approved by the Ethics Committee of the NIGRR and all procedures were performed in accordance with the ethical standards and with the 1964 Helsinki Declaration and its later amendments.

The diagnosis of SS was established according to the American-European Consensus Group classification criteria [14]. Characteristics of patients are summarised in Table I. Clinical evaluation of patients was based on medical history, physical examination and basic diagnostic laboratory tests. The patients were grouped under the following serological profiles: 1) negative for anti-SSA/Ro and anti-SSB/La antibodies, 2) positive for anti-SSA/Ro antibody only, and 3) seropositive for both anti-SSA/Ro and anti-SSB/La antibodies.

Sera were isolated by routine laboratory methods and samples were stored in aliquots at $-70^{\circ} \mathrm{C}$ until assayed. In sera samples the presence of various autoantibodies was tested and the concentrations of select cytokines were measured. The presence and titres of the ANAs were determined using an indirect immunofluorescence method with the Hep2 cell line as an antigen, anti-Ro/SSA and anti-La/SSB were detected using enzyme immunoassay (ELiA IgG test Unicap 100 Phadia GmbH), while RF of IgM class (normal range $<34 \mathrm{IU} / \mathrm{ml}$ ) and other autoantibodies were identified by routine laboratory tests. The concentrations of cytokines were measured using commercially available enzyme-linked immunosorbent assay (ELISA) kits specific for humans: 1) IL-4, IL-10, TSLP (Ready-SET-Go kits) and APRIL (Platinum ELISA kit) from eBioscience, An Affymetrix Company, San Diego, CA, USA; 2) BAFF (Quantikine kit) from R\&D Systems Europe, Abingdon, UK; 3) IFN- $\alpha$ (Multi-subtype ELISA kit) from PBL Assay Science, Piscataway, NJ, USA.

Data were analysed using Prism 5, GraphPad Software Inc., San Diego, CA, USA. For intergroup comparison of continuous and discrete variables the Mann-Whitney $U$ and Fisher's exact tests were applied, respectively. Correlation was assessed using Spearman's rank test; $p$-values $<0.05$ were considered significant.

\section{Results}

As shown in Table I, the majority of our patients suffered from the primary form of the syndrome and rheumatoid arthritis predominated over other concomitant autoimmune diseases in patients with the secondary form of SS. A greater number of patients were female and the cohort was heterogeneous for there were wide variations in patients' age, age at diagnosis and disease duration. The majority of patients were treated with antimalarial drugs or glucocorticoids. We failed to find any significant differences in analysed parameters between pSS and sSS patients or between female and male patients (not shown). The only exception was longer disease duration in sSS patients $(\times \pm$ SEM $=121 \pm 22$ vs. $54 \pm 12$ months; $p=0.004)$ and more frequent joint involvement (94\% vs. $69.8 \%$; $p=$ 0.00005), as compared with pSS patients.

For further analysis the patients were categorised according to the absence (group $1 ; n=15$ ) or the presence of anti-SSA/Ro autoantibody, which occurred either alone (group 2; $n=27$ ) or concomitantly with anti-SSB/La autoantibody (group 3; $n=39$ ). The secondary form of SS was diagnosed in one-third of patients of groups 1 and 2 (33.3\% and 29.6\%, respectively) and was less frequent (13\%) in patients assigned to group 3. Comparison of the patients' subgroups revealed that they also differed in: 1) the frequency of occurrence of other autoantibodies, including RF, 2) the number of autoantibodies of various specificities, and 3) the titre of ANA, which were all higher in group 2 and/or 3 than in group 1. In addition, diversity of autoantibodies and the frequency of RF positivity were higher in group 3 than group 2 of patients (Fig. 1).

As shown in Figure 2, patients of groups 2 and 3 were characterised by significantly earlier disease onset than the patients of group 1, while there was no difference in the median disease duration between groups. Similarly to differences in the age of SS onset, patients of group 1 were older than the rest of subjects. When compared to healthy volunteer controls, patients of group 1 and, to a lesser degree, of group 2 were older, while patients of group 3 were of similar age. Because of these differences, we additionally analysed the relationships between the age of study participants and tested variables, i.e. serum concentrations of cytokines and presence of autoantibodies, using Spearman's rank test. In both healthy volunteers and SS patients we failed to find any significant correlations between the age and serum concentrations of cytokines, because $R$ coefficient values were within the range 0.02-0.2 and associations did not reach statistical significance ( $p$-values within 0.9-0.1; data not shown in figures). However, a statistically significant weak inverse correlation was found for age and anti-SSA/Ro $(R=-0.31 ; p=0.005)$ and anti-SSB/La $(R=$ $-0.295 ; p=0.008)$ positivity in the entire patient group (not shown in figures).

Regarding clinical symptoms, the patients of group 2 and/or 3 were characterised by higher frequency of the positive Schirmer's test ( $\leq 5 \mathrm{~mm} / \mathrm{min}$ ) and skin involvement, comprising xeroderma, cutaneous vasculitis (palpable and nonpalpable purpura), Raynaud's syndrome 
A

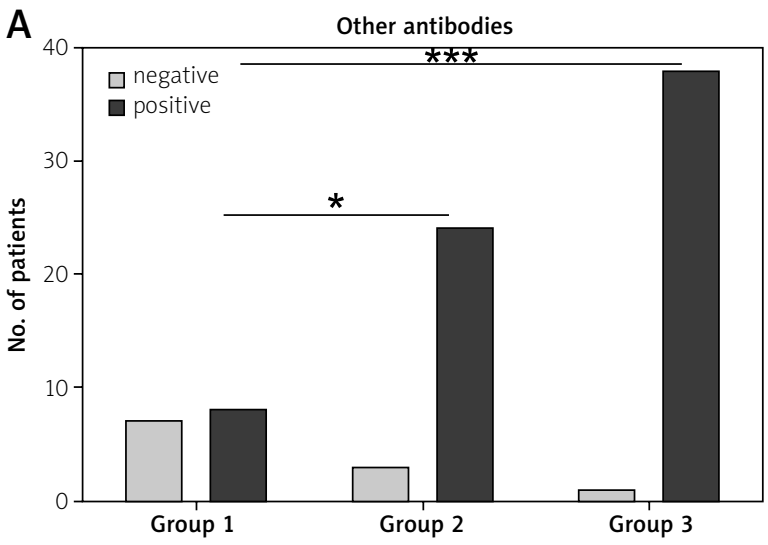

C

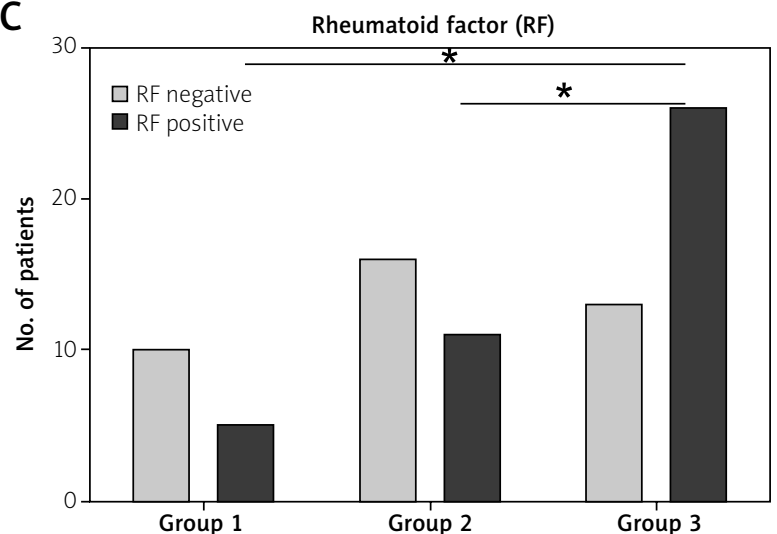

B

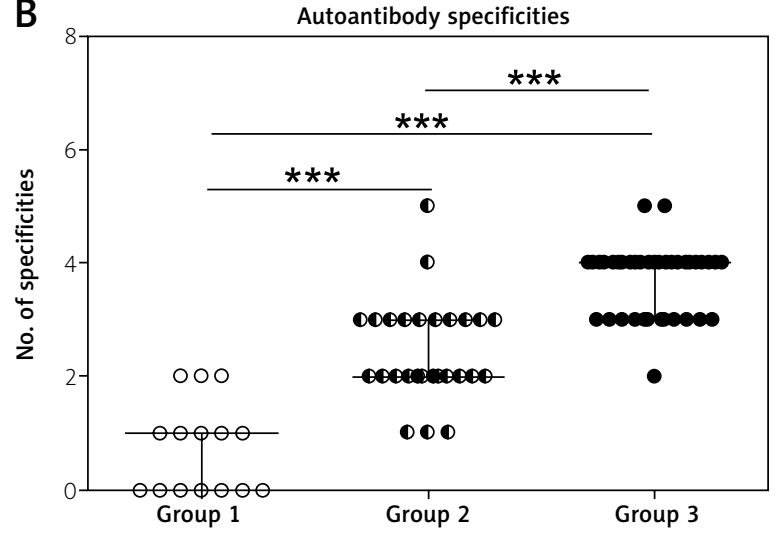

D

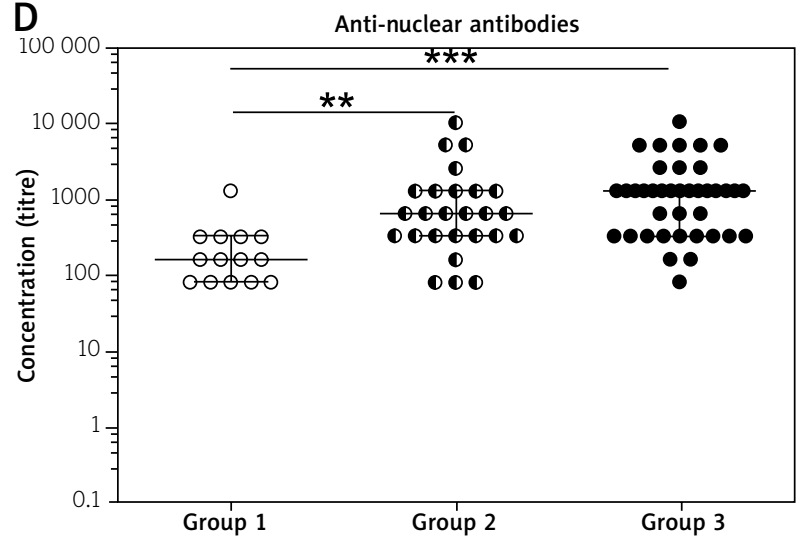

Fig. 1. Serological differences between subgroups of Sjögren's syndrome patients. Data are expressed as the median with interquartile range (IQR) of continuous variables (panels $B$ and $D$ ) or as a frequency distribution of discrete variables (panels $A$ and $C$ ). Patients were categorised as follows: group 1 - patients negative for anti-SSA/Ro and anti-SSB/La antibodies, group 2 - patients positive for anti-SSA/Ro antibody only, and group 3 - patients seropositive for both anti-SSA/Ro and anti-SSB/La antibodies. The number of patients having autoantibodies other than anti-SSA/Ro and anti-SSB/La (A), number of specificities of autoantibodies (B), number of patients positive for rheumatoid factor (RF) (C) and the titre of antinuclear antibodies ( $D$ ) are shown in every group. Each point represents one patient ( $B$ and $D$ ). For statistically significant differences between patient groups $p$-values are shown: ${ }^{\star} p=0.05-0.01 ;{ }^{* \star} p=0.01-0.001 ;{ }^{* \star *} p<0.0001$.

and/or urticarial-like lesions, eyelid dermatitis, and pruritus, compared with group 1 patients (Fig. 3). As for tested factors, serum concentrations of APRIL were higher in the patients of both groups 2 and 3 than those of group 1 and healthy volunteers, but there was no significant difference between cohorts in the serum levels of all other cytokines (Fig. 4).

\section{Discussion}

Our patient cohort was heterogeneous with respect to demographic data. Despite this, clinical symptoms were typical and similar to manifestations described by others. Also the frequency of occurrence of disease-related autoantibodies (Table I) was much the same [8].
In accordance with the findings of other authors [5] we observed that about half of patients (48.2\%) had both anti-SSA/Ro and anti-SSB/La autoantibodies (group 3), in about one third (33.3\%) anti-SSA/Ro but not anti-SSA/La autoantibodies were present (group 2), while the remaining patients $(18.5 \%)$ were negative for autoantibodies of these specificities (group 1). However, in every group other SS-related autoantibodies were also found and the frequency of their occurrence, including RF, was the lowest in group 1 (Fig. 1, panels A and C). Consistently, the number of autoantibodies of different specificities was the lowest in group 1, higher in group 2 and the highest in group 3 (Fig. 1, panel B). Thus, by showing that the presence of anti-SSA/Ro antibodies is associated with a more 

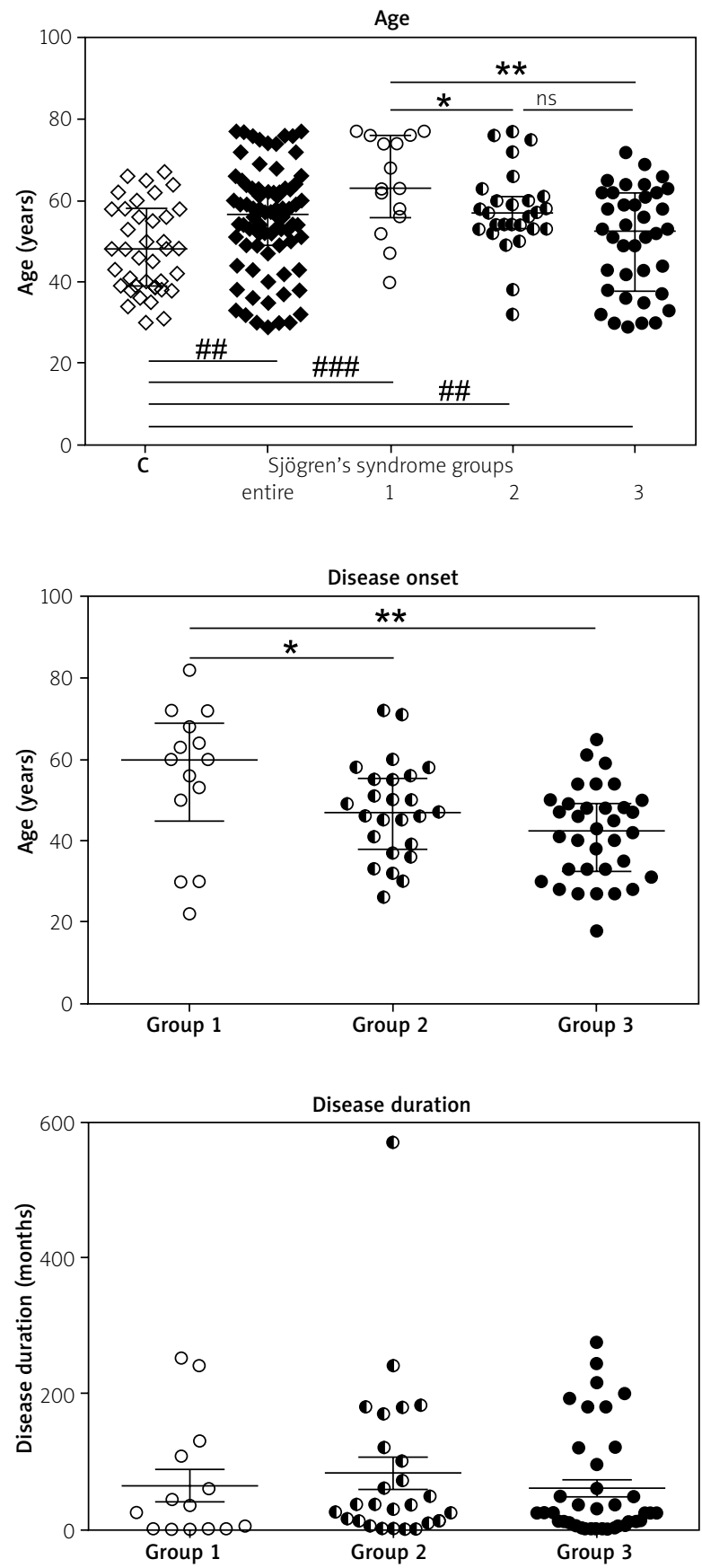

Fig. 2. Demographic differences between subgroups of Sjögren's syndrome patients. Data are expressed as the median with interquartile range $(\mathrm{IQR})$. Each point represents one patient or healthy volunteer. Patients were categorised as described in Figure 1. For statistically significant differences between patients and healthy control (C) ${ }^{\# \# p} p=$ $\left.0.01-0.001 ;{ }^{\# \#} p<0.0001\right)$ as well as between patient subgroups ( $\left.{ }^{*} p=0.05-0.01 ;{ }^{* *} p=0.01-0.001\right)$ $p$-values are shown; ns - not significant. diversified pattern of autoantibodies (Fig. 1, panels A-C) and higher titre of ANA (Fig. 1, panel D), our findings give support for the occurrence of "epitope spreading" of the autoimmune humoral response in these patients. Interestingly, concomitant positivity for anti-SSB/La antibodies has an additional enhancing effect, at least in some aspects (Fig. 1, panels B and C).

It is well known that increasing diversity of antibodies reflects the physiological phenomenon named "B cell epitope spreading" which is an antigen-driven process leading to diversification of the epitopes recognised by the immune system and thus to maturation of the humoral response [15]. Although epitope spreading efficiently improves clearing of various infectious agents, it may be harmful when autoantigens are the targets of immune attack. In animal models of SS, immunization of mice with Ro60 peptides results in epitope spreading with the formation of both anti-Ro and anti-La antibodies, lymphocytic infiltration and functional impairment of salivary glands, similar to human SS [16]. However, in patients with established SS it is difficult to determine the sequence of events that results in an immune response against a variety of autoantigens. Also the contribution of autoantibodies to SS pathogenesis is unclear. Despite this, numerous data show the link between presence of particular autoantibodies and clinical symptoms. For example, anti-SSA/Ro and anti-SSB/La antibodies have been reported to be associated with an early disease onset, glandular dysfunction, extra-glandular manifestations, B cell activation and high risk of lymphadenopathy, while atypical autoantibodies such as those specific to phospholipids, centromeres or interferon-inducible protein-16 are related to immunological diseases (e.g. thyroiditis), more severe exocrine glandular dysfunction or more severe underlying disease, respectively [17-20].

Conversely, pSS patients negative for anti-SSA/Ro and anti-SSB/La antibodies have been characterised as having low risk of lymphoma and low level of B cell expansion [21]. On the other hand, comparison of pSS patients with an early and late disease onset that occurred before or after the age of 35, respectively, has also shown an association between young onset, anti-SSA/Ro and RF positivity and more severe clinical symptoms, including a higher incidence of lymphomas [22]. The present results, showing that SS patients with a more diversified autoimmune humoral response (groups 2 and 3) develop clinical symptoms at a younger age than patients with a more restricted (in terms of both quantity and quality) autoantibody profile, devoid of both anti-SSA/ Ro and anti-SSB/La antibodies (group 1; Fig. 2), are consistent with the above observations of other authors $[17,18,22]$. In this regard, we have also found that the presence of anti-SSA/Ro is more important because in 

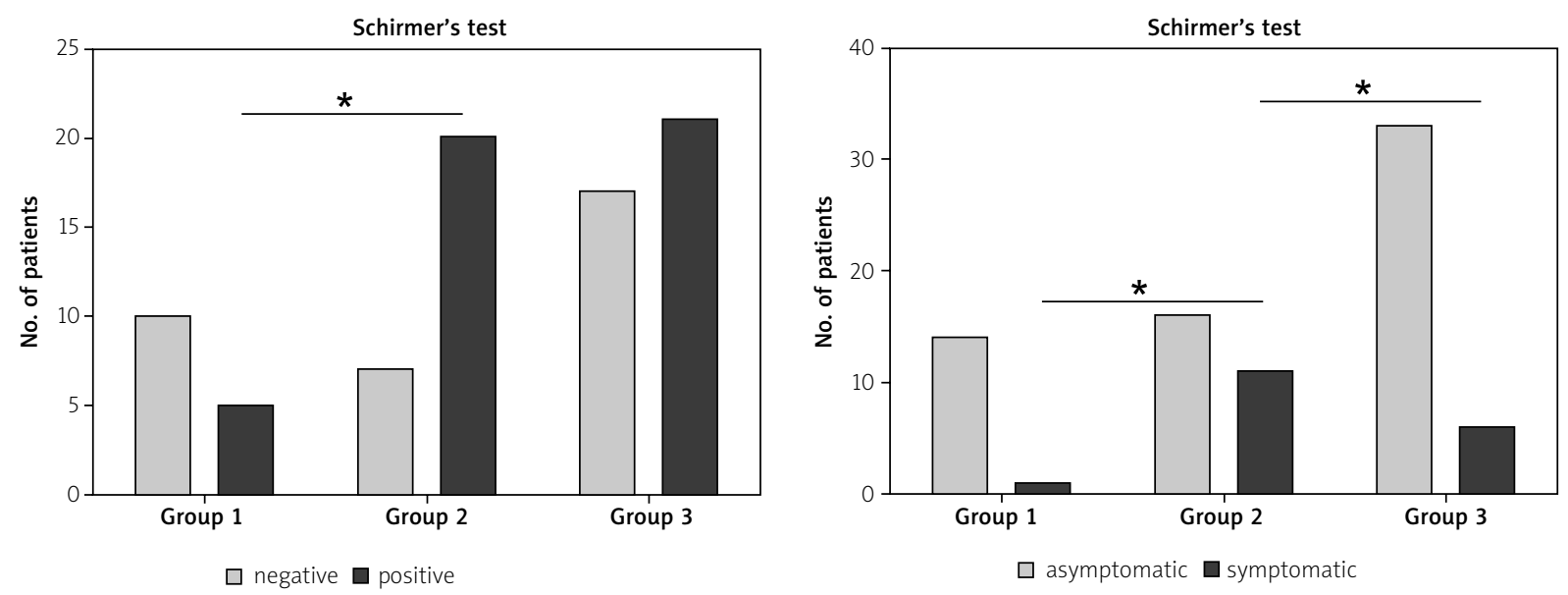

Fig. 3. Clinical differences between subgroups of Sjögren's syndrome patients. Data are expressed as a frequency distribution of discrete variables. Patients were categorised as described in Figure 1 . For statistically significant differences between patient subgroups $p$-values are shown: ${ }^{*} p=0.05-0.01$.

the group of patients with co-existing anti-SSA/Ro and anti-SSB/La antibodies the age of SS onset is only slightly diminished, even though each of the two antibodies inversely correlates with it (see Results).

Interestingly, we noted that patients from subgroup 2 (anti-SSA/Ro positive) more often have a positive Schirmer's test and skin involvement than other patients. In addition, the co-existence of anti-SSA/Ro and anti-SSB/La antibodies (subgroup 3) even has some protective effect, especially in relation to skin symptoms (Fig. 3). This again suggests a stronger relationship of anti-SSA/Ro than anti-SSB/La with both lacrimal gland dysfunction and development of skin lesions. Other authors have previously reported that skin lesions in pSS, especially purpura and oedematous annular erythema, are associated with anti-SSA/Ro and anti-SSB/La antibodies and thus suggested a role of these antibodies in the development of these cutaneous lesions [23, 24].

Finally, we analysed the serum levels of several cytokines relevant to SS pathogenesis in our patient subgroups and found that only concentrations of APRIL, but not BAFF and other tested factors, were significantly elevated in anti-SSA/Ro positive patients (group 2), compared to healthy control and anti-Ro/La negative patients. Moreover, in the patients positive for both anti-SSA/Ro and anti-SSB/La antibodies (group 3) there was some tendency to a further rise of APRIL level but the difference did not reach statistical significance (Fig. 4). Thus, our results suggest an association of systemic APRIL up-regulation with humoral response characterised by more intense epitope spreading and presence of primarily anti-SSA/Ro antibody. BAFF and APRIL are cytokines of the TNF family and have both overlapping and distinct roles in humoral immunity. BAFF is critical for the differentiation of imma- ture to mature B cells while APRIL acts at a later stage, modulating the function and survival of antigen-experienced B cells and maintaining the plasma cell pool [25].

In SS patients raised serum levels of both BAFF and APRIL have been found although there are also data showing no difference in BAFF levels between patients and healthy volunteers [25-27]. Some authors have reported the relationships between circulating concentrations of these cytokines and disease activity [27, 28]. Although these findings are somewhat inconsistent, both cytokines are thought to contribute to SS pathogenesis. The present results showing up-regulation of the circulating pool of APRIL and its association with a more diversified autoantibody response suggest that this cytokine may represent a therapeutic target for the treatment of SS patients characterised by early disease onset, epitope spreading, presence of anti-SSA/Ro, xerophthalmia and/ or skin involvement (groups 2 and 3). This is an important observation because to date therapeutic strategies for SS are mainly symptomatic and not efficient enough in modifying the disease course. Moreover, conducted clinical studies using biological drugs, i.e. B cells depleting rituximab and BAFF neutralising belimumab, were not unanimous in reaching the primary efficacy endpoints [2, 3, 29].

\section{Conclusions}

In summary, we report that the SS patient subgroup with a more diversified autoimmune humoral response and with the presence of anti-SSA/Ro antibody is characterised by disease onset at younger age, higher frequency of xerophthalmia, skin involvement and up-regulated serum concentration of APRIL - a cytokine which may represent a therapeutic target for these patients. 

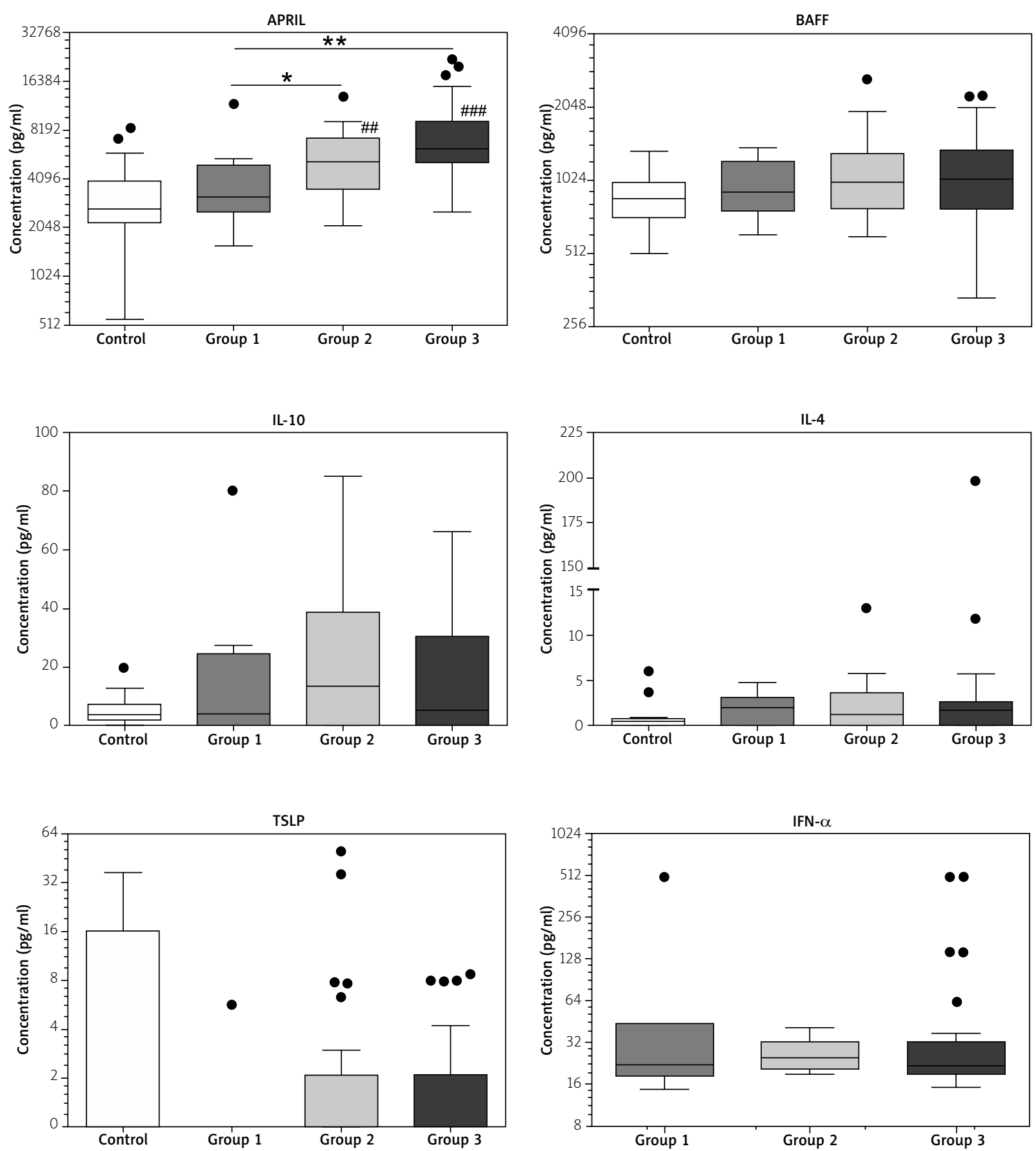

Fig. 4. Serum concentrations of tested cytokines in subgroups of Sjögren's syndrome patients. Results are expressed as the median (horizontal line) with interquartile range (IQR, box), lower and upper whiskers (data within 3/2xIQR) and outliers (points) (Tukey's box). Patients were categorised as described in Figure 1. Control - healthy volunteers; APRIL - a proliferation inducing ligand; BAFF - B-lymphocyte activating factor; IL - interleukin; TSLP - thymic stromal lymphopoietin; IFN- $\alpha$ - interferon $\alpha$. For statistically significant differences between patients and healthy control (\#\# $p=0.01-0.001$; $\left.{ }^{\# \#} p<0.0001\right)$ as well as between patient subgroups $\left({ }^{*} p=0.05-0.01 ;{ }^{* *} p=0.01-0.001\right) p$-values are shown. 


\section{Acknowledgements}

We gratefully acknowledge all patients and healthy volunteers for their contribution to the study.

This study was funded by the National Institute of Geriatrics, Rheumatology and Rehabilitation (grant number S/8), Warsaw, Poland. The National Institute of Geriatrics, Rheumatology and Rehabilitation is supported by a core grant from the Ministry of Science and Higher Education (Poland).

The authors declare no conflict of interest.

\section{References}

1. Brito-Zeron P, Baldini C, Bootsma H, et al. Sjögren syndrome. Nat Rev Dis Primers 2016; 2: 16047.

2. Barone F, Colafrancesco S. Sjögren syndrome: from pathogenesis to novel therapeutic targets. Clin Exp Rheumatol 2016; 34 (Suppl 98): S58-S62.

3. Ferro F, Marcucci E, Orlandi M, et al. One year in review 2017: primary Sjögren's syndrome. Clin Exp Rheumatol 2017; 35: 179-191.

4. Shiboski CH, Shiboski SC, Seror R, et al. 2016 American College of Rheumatology/European League Against Rheumatism classification criteria for primary Sjögren's syndrome: a consensus and data-driven methodology involving three international patient cohorts. Ann Rheum Dis 2017; 76: 9-16.

5. Vasiliki-Kalliopi B, Vlachoyiannopoulos PG. Subgroups of Sjögren syndrome patients according to serological profiles. J Autoimmunity 2012; 39: 15-26.

6. Routsias JG, Tzioufas AG. Autoimmune response and target autoantigens in Sjögren's syndrome. Eur J Clin Invest 2010; 40: 10261036.

7. Theander E, Jonsson R, Sjöström B, et al. Prediction of Sjögren's syndrome years before diagnosis and identification of patients with early onset and severe disease course by autoantobody profiling. Arthritis Rheum 2015; 67: 2427-2436.

8. Baldini C, Pepe P, Quartuccio L, et al. Primary Sjögren's syndrome as a multi-organ disease: impact of the serological profile on the clinical presentation of the disease in a large cohort of Italian patients. Rheumatology (Oxford) 2014; 53: 839-844.

9. Szodoray P, Jonsson R. The BAFF/APRIL system in systemic autoimmune diseases with a special emphasis on Sjögren's syndrome. Scand J Immunol 2005; 62: 421-428.

10. Youinou P, Pers JO. Disturbance of cytokine networks in Sjögren's syndrome. Arthritis Res Ther 2011; 13: 227.

11. Roeschler N, Tak PP, Illei GG. Cytokines in Sjögren's syndrome: potential therapeutic targets. Ann Rheum Dis 2010; 69: 945-948.

12. Hillen MR, Kruize AA, Bikker A, et al. Decreased expression of thymic stromal lymphopoietin in salivary glands of patients with primary Sjögren's syndrome is associated with increased disease activity. Mod Rheumatol 2016; 26: 105-109.

13. Bodewes ILA, Al-Ali S, van Helden-Meeuwsen CG, et al. Systemic interferon type I and type II signatures in primary Sjögren's syndrome reveal differences in biological disease activity. Rheumatology (Oxford) 2018; 57: 921-930.
14. Vitali C, Bombardieri S, Jonsson R, et al. Classification criteria for Sjögren's syndrome: a revised version of the European criteria proposed by the American-European Consensus Group. Ann Rheum Dis 2002; 61: 554-558.

15. Cornaby C, Gibbson L, Mayhew V, et al. B cell epitope spreading: mechanisms and contribution to autoimmune diseases. Immunol Lett 2015; 163: 56-68.

16. Kurien BT, Dsouza A, Igoe A, et al. Immunization with 60 kD Ro peptide produces different stages of preclinical autoimmunity in a Sjögren's syndrome model among multiple strains of inbred mice. Clin Exp Immunol 2013; 173: 67-75.

17. Hernández-Molina G, Leal-Alegre G, Michel-Peregrina $M$. The meaning of anti-Ro and anti-La antibodies in primary Sjögren's syndrome. Autoimmunity Rev 2011; 10: 123-125.

18. Garcia-Carrasco M, Mendoza-Pinto C, Jiménez-Hernández C, et al. Serological features of primary Sjögren's syndrome: clinical prognostic correlation. Int I Clin Rheumatol 2012; 7: 651-659.

19. Baer AN, Medrano L, McAdams-DeMarco M, et al. Association of anticentromere antibodies with more severe exocrine glandular dysfunction in Sjögren's syndrome: analysis of the Sjögren's International Collaborative clinical alliance cohort. Arthritis Care Res 2016; 68: 1554-1559.

20. Baer AN, Petri M, Sohn J, et al. Association of antibodies to interferon-inducible protein-16 with markers of more severe disease in primary Sjögren's syndrome. Arthritis Care Res 2016; 68: 254-260.

21. Quartuccio L, Baldini C, Bartoloni E, et al. Anti-SSA/SSB-negative Sjögren's syndrome shows a lower prevalence of lymphoproliferative manifestations, and a lower risk of lymphoma evolution. Autoimmunity Rev 2015; 14: 1019-1022.

22. Ramos-Casals M, Cervera R, Font J, et al. Young onset of primary Sjögren's syndrome: clinical and immunological characteristics. Lupus 1998; 7: 202-206.

23. Nishiyama S, Miyawaki S, Hashimoto T. Cutaneous manifestations of primary Sjögren's syndrome. Nihon Rinsho 1995; 53: 25512556.

24. Brito-Zeron P, Retamozo S, Akasbi M, et al. Annular erythema in primary Sjögren's syndrome: description of non-Asian cases. Lupus 2014; 23: 166-175.

25. Samy E, Wax S, Huard B, et al. Targeting BAFF and APRIL in systemic lupus erythematosus and other antibody-associated diseases. Int Rev Immunol 2017; 36: 3-19.

26. Vosters JL, Roescher N, Polling EJ, et al. The expression of APRIL in Sjögren's syndrome: aberrant expression of APRIL in the salivary gland. Rheumatology (Oxford) 2012; 51: 1557-1562.

27. Deng F, Chen J, Zheng J, et al. Association of BAFF and IL-17A with subphenotypes of primary Sjögren's syndrome. Int J Rheum Dis 2016; 19: 715-720.

28. Jonsson MV, Szodoray P, Jellestad S, et al. Association between circulating levels of the novel TNF family members APRIL and BAFF and lymphoid organization in primary Sjögren's syndrome. J Clin Immunol 2005; 25: 189-201.

29. Bootsma H, Kroese FGM, Vissink A. Rituximab in the treatment of Sjögren's syndrome: is it the right or wrong drug? Arthritis Rheumatol 2017; 69: 1346-1349. 